\title{
Influence sur les infections mammaires du giclage avant et après la traite ${ }^{(*)}$
}

\author{
par \\ M. ROGUINSKY \\ (Collaboration technique : F. BARRAULT, R. BORDE, M. DUCELLIEZ) \\ Institut national de la Recherche agronomique \\ Station de Pathologie de la Reproduction. Centre de Tours \\ 37380 Nouzilly
}

La transmission des infections mammaires de la vache s'effectue de quartier infecté à quartier sain pendant la traite ; les bactéries sont déposées sur l'extrémité du trayon et dans le canal strié (Newbould, 1964) et n'envahissent le sinus galactophore qu'entre les traites (Neave, Olivier et Dodd, 1957).

Phillips a proposé en 1969 une méthode particulière d'extraction des premiers jets de lait (qu'il désigne sous le nom de "squirting ") pour éliminer les bactéries déposées dans le canal du trayon. Ce "squirting " ou en français " giclage " doit être réalisé avant toute manipulation de la mamelle (en pratique avant le lavage) de façon à éviter une dispersion dans le sinus galactophore des bactéries présentes dans le canal strié. Cette précaution différencie le giclage de deux techniques assez répandues, effectuées après lavage, l'élimination des premiers jets de lait considérés comme les plus chargés en germe et leur projection sur un bol de traite pour déceler précocement les mammites cliniques. Phillips a proposé par la suite que ce giclage soit effectué après la traite, pour permettre l'élimination des germes déposés dans le canal avant leur multiplication. D'après ces essais les deux méthodes permettent une réduction du nombre des nouvelles infections (Frost et Phillips, 1970 ; Phillips, 1969 ; Phillips, Whiteman et Walker, 1969).

Une autre méthode de prévention des infections, le trempage des trayons après la traite dans une solution désinfectante, est acceptée par de nombreux éleveurs et son efficacité a déjà été bien démontrée (Feagan, Hehir et White, 1970 ; Neave, Dodd et Kingwill, 1966 ; Schultze, 1969). Si la supériorité du giclage peut être mise

(*) Bull. Acad. Vét. de France, 1973, t. 46, n 5, 239. 
en évidence par rapport au trempage et comme il est a priori aussi simple et moins onéreux, son application peut être envisagée. Cette technique, facile à mettre en œuvre, représenterait un progrès dans la prophylaxie des infections mammaires, tout en apportant une compréhension accrue du mécanisme de pénétration des bactéries ; mais elle constitue quand même une opération supplémentaire pour le trayeur et son bilan sanitaire et économique doit être examiné de près.

Nous avons réalisé sur notre troupeau des expériences successives sur deux lactations, en comparant essentiellement le taux de nouvelles infections (nombre d'infections par quartier apparues dans la période considérée) des lots témoins et des lots soumis au giclage, au trempage ou aux deux. Les animaux sont répartis par tirage au sort entre les lots et placés dans l'étable en alternant les vaches de chaque lot pour que les risques de contamination soient comparables. Dans les deux expériences, l'observation des infections est réalisée au $15^{\mathrm{e}}$ jour après le vêlage jusqu'à la fin de la lactation.

La première expérience comprend 10 vaches par lot; chaque lot est soumis à l'un des traitements suivants :

- Lot Témoin : aucun traitement ;

- Lot Giclage après la traite : giclage suivant la technique de Phillips (1969). Le haut du trayon est saisi entre le pouce et l'index d'une main, un jet de lait est extrait à la pincée et le haut du trayon est repris par le pouce et l'index de l'autre main alors que le bas du trayon est encore tenu par la première main. Trois jets de lait sont ainsi extraits de chaque quartier ;

- Lot Trempage : trempage des trayons après la traite dans une solution d'iodophore à 5000 ppm d'iode (Iobac, Roger Bellon) ;

- Lot Giclage et trempage : effectués après la traite dans l'ordre giclage puis trempage.

La deuxième expérience comprend 17 vaches par lot, soumises à l'un des traitements suivants :

- Lot Témoin : aucun traitement ;

- Lot Giclage avant la traite : giclage suivant la méthode de Phillips (1969) avant la traite, avant le nettoyage de la mamelle et avant tout prélèvement. Toutes les manipulations ou stimulations de la mamelle sont ainsi évitées (pour empêcher dans la mesure du possible une dispersion des germes vers le sinus galactophore). Trois jets sont extraits de chaque quartier.

La traite a lieu en place à l'étable, avec un système de transfert du lait dans un lactoduc (Alfamatic, Alfa-Laval). Le tarissement est pratiqué par arrêt brusque de la traite 2 mois avant vêlage ou lorsque la production devient inférieure à 51 par jour. Des prélèvements de lait de début de traite sont effectués toutes les 2 semaines pour examen bactériologique sur gélose au sang-esculine (Plommet, 1962). Un quartier est considéré comme infecté soit 
lorsque le même germe pathogène est isolé à partir de deux ou plusieurs prélèvements successifs, soit lorsqu'un germe pathogène est isolé d'un échantillon provenant d'un quartier qui présente des signes cliniques.

Nous avons obtenu les résultats suivants pour la première expérience : 41 p. 100 ;

- Lot Témoin : 15 infections nouvelles pour 37 quartiers, soit

- Lot Giclage après la traite : 1 pour 40 quartiers, soit 2,5 p. 100 ;

- Lot Trempage : 5 pour 39 quartiers, soit 12 p. 100 ;

- Lot Giclage et Trempage : 6 pour 39 quartiers, soit 15 p. 100.

Les différences entre le lot Témoin et le lot Giclage sont hautement significatives $(\mathrm{P}<0,005)$; celles entre le lot Témoin et les lots Trempage ou Giclage et Trempage sont significatives $(P<0,05)$. Les différences entre le lot Giclage et les lots Trempage ou Giclage et Trempage ne sont pas significatives $(\mathrm{P}>0,1)$.

Pour la deuxième expérience les résultats sont les suivants : 13 p. 100 ;

- Lot Témoin : 9 infections nouvelles pour 67 quartiers, soit

- Lot Giclage avant la traite : 8 infections nouvelles pour 68 quartiers, soit 12 p. 100.

Aucune différence significative entre les deux lots ne peut être mise en évidence dans cette expérience. Le faible taux de nouvelles infections dans le lot Témoin de la deuxième expérience par rapport à la première est vraisemblablement dû à des essais de vaccination et de traitements effectués au tarissement sur la plus grande partie de l'effectif entre les deux expériences successives.

Dans les deux expériences, toutes les infections nouvelles sont dues à des Streptococceae ou à des staphylocoques, sauf une due à un bacille.

Les résultats obtenus avec le giclage après la traite confirment les résultats de Phillips (1969) sur la diminution du nombre d'infections mammaires par cette méthode. Par contre ceux du giclage avant la traite sont en contradiction avec les résultats de Phillips, Whiteman et Walker (1969) et de Frost et Phillips (1970), mais en accord avec ceux de Feagan et Hehir (1972). Il faut noter que leurs conditions expérimentales sont différentes des nôtres ; ainsi Phillips et al. opèrent soit par comparaison des quartiers droits et gauches et isolement des germes pathogènes à partir des seuls échantillons à CMT positifs, soit par l'étude de l'infection expérimentale suivie ou non du giclage.

D'un point de vue pathogénique, la différence entre giclage avant et après la traite indique que la multiplication des germes dans le canal du trayon dans le temps qui sépare deux traites est importante ; à ce moment l'élimination par l'effet de rinçage suggéré 
par Phillips est trop partielle pour être efficace si on pratique le giclage avant la traite suivante. On peut aussi penser, comme Forbes (1968) l'avait observé dans l'infection expérimentale, que les bactéries, en se multipliant rapidement, envahissent le sinus galactophore d'où elles ne peuvent plus être chassées par la traite suivante ou le giclage.

Pour le trempage, nous retrouvons la diminution des infections nouvelles signalées par d'autres auteurs (Feagan, Hehir et White, 1970 ; Neave, Dodd et Kingwill, 1966 ; Schultze, 1969). Cette diminution est apparemment moindre que pour le giclage. De plus, il est intéressant de constater que l'association du gliclage et du trempage ne modifie pas les résultats obtenus, ce qui indique que ces deux procédés agissent par le même mécanisme ; ils éliminent physiquement ou chimiquement les germes présents sur l'extrémité ou dans le canal du trayon.

En conclusion, nous pouvons déjà préconiser le giclage après la traite comme méthode diminuant le nombre des infections nouvelles. Son efficacité semble supérieure à celle du trempage et son coût est nettement moins élevé puisqu'aucun produit ne doit être utilisé ; toutefois le temps moyen par vache nécessité par le giclage (14 s) est supérieur à celui demandé par le trempage $(9 \mathrm{~s})$. Le trempage ne devrait être maintenu que dans le cas de plaies nombreuses du trayon, qui peuvent être cicatrisées par l'action du produit (glycérine ou lanoline) associé à l'iodophore. Nous avons voulu, cependant, vérifier les bons résultats obtenus lors de la première expérience pour le giclage sur un plus grand nombre d'animaux ; c'est pourquoi une expérience est en cours sur notre troupeau expérimental comparant l'évolution du nombre d'infections de 20 vaches «Témoin » et de 20 vaches Giclage après traite pendant toute leur lactation.

\section{Bibliographie}

1. Feagan (J. T.), Hehir (A. F.). - Austr. vet. J., 1972, 48, 664-667.

2. Feagean (J. T.), Hehir (A. F.), Whitte (B. R.). - Austr. J. Dairy Technol., $1970,25,87-90$.

3. Forbes (D.). - J, Dairy Res., 1968, 35, 399-406.

4. Frost (A. J.), Phillips (D. S. M.). - Veter. Rec., 1970, 86, 592-595.

5. Neave (F. K.), Dodd (F. H.), Kingwill (R. G.). - Veter. Rec., 1966, 78, 521-523.

6. Neave (F. K.), Oliver (J.), Dodd (F.H.). - Rep. nat. Inst. Res. Dairy, Reading, 1957.

7. Newbould (F. H. S.). - Dairy Sci. Abstr., 1964, 26, 245-255.

8. Phillips (D. S. M.). - Proceed. Ruakura Farm. Conf. week, 1969.

9. Phillips (D. S. M.), Whiteman (D. P.), Walker (H. T. M.). - New Z. Veter. J., $1969,17,90-91$.

10. Plommet (M.). - Ann. Nutrit. Aliment. Fr., 1962, 16, 287-316.

11. Schultze (W. D.). - J. Dairy Sci., 1969, 53, 38-45. 